Rhode Island College

Digital Commons @ RIC

\title{
The Relationship Between Osteoarthritis, Hip Arthroplasty, and Post-Operative Ambulation Distances
}

\author{
Melissa E. Ings \\ Rhode Island College
}

Follow this and additional works at: https://digitalcommons.ric.edu/etd

Part of the Other Nursing Commons

\section{Recommended Citation}

Ings, Melissa E., "The Relationship Between Osteoarthritis, Hip Arthroplasty, and Post-Operative Ambulation Distances" (2015). Master's Theses, Dissertations, Graduate Research and Major Papers Overview. 118.

https://digitalcommons.ric.edu/etd/118

This Major Paper is brought to you for free and open access by the Master's Theses, Dissertations, Graduate Research and Major Papers at Digital Commons @ RIC. It has been accepted for inclusion in Master's Theses, Dissertations, Graduate Research and Major Papers Overview by an authorized administrator of Digital Commons @ RIC. For more information, please contact digitalcommons@ric.edu. 
THE RELATIONSHIP BETWEEN OSTEOARTHRITIS, HIP ARTHROPLASTY, AND POST-OPERATIVE AMBULATION DISTANCES

A Major Paper Presented

By

Melissa E. Ings

Approved:

Committee Chairperson

Committee Members

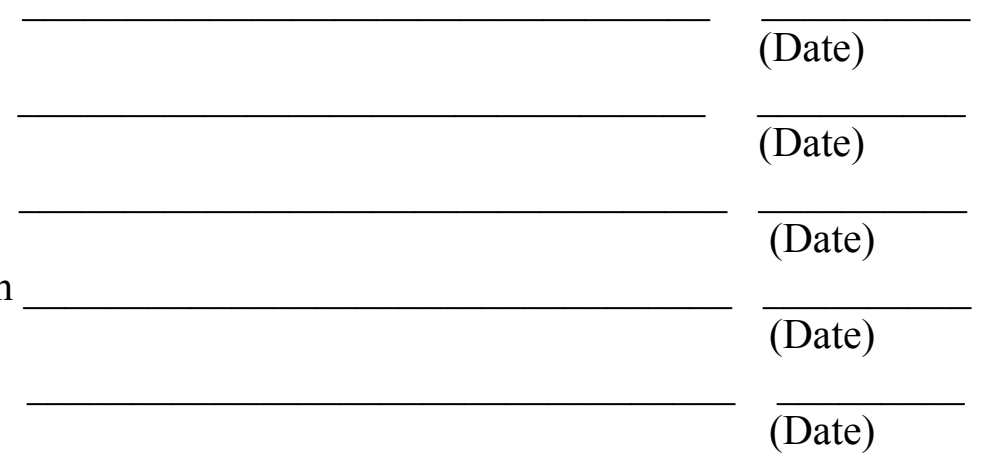

Director of Master's Program

Dean, School of Nursing

(Date) 
THE RELATIONSHIP BETWEEN OSTEOARTHRITIS, HIP ARTHROPLASTY, AND POST-OPERATIVE AMBULATION DISTANCES

by

Melissa E. Ings

A Major Paper Submitted in Partial Fulfillment

of the Requirements for the Degree of

Master of Science in Nursing

In

The School of Nursing

Rhode Island College

2015 


\begin{abstract}
Osteoarthritis, total hip replacements, and post-operative ambulation distances are three interrelated concepts that lead patients from diagnosis, to intervention, and to the first indications of recovery post-operatively. The purpose of the project was to quantify the ambulation distances of patients with one of three surgical approaches for total hip replacements (THR). The timeframe for measurement was from the day of surgery (day 0) through the second post-operative day (POD 2) comparing different surgical approaches with length of ambulation distances. Pre-operative elective joint replacement educational classes, may play a role in post-operative recovery, which provided the rationale for including them in this project. Electronic health records of 20 patients from each of the three surgical approaches (anterior, posterior, and lateral), were retrieved; data regarding the ambulation distances were averaged for each surgical approach. The results revealed no advantage, in terms of average ambulation distance, of one surgical approach over another. All members of the healthcare team strive to provide patientcentered, quality care in the most fiscally responsible manner. Increasing post-operative ambulation distances after a THR may contribute to earlier discharge and economic benefit to the healthcare system. A replication study with a larger sample may yield additional findings and guide the advanced nurse practitioner in developing an evidencebased approach to post-operative care of THR patients.
\end{abstract}




\section{Table of Contents}

Table of Contents $\quad$ Page

Statement of Problem.............................................................

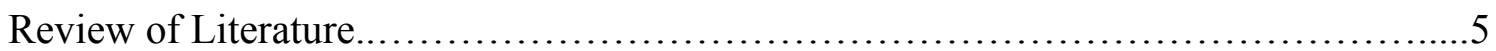

Theoretical Framework.....................................................25

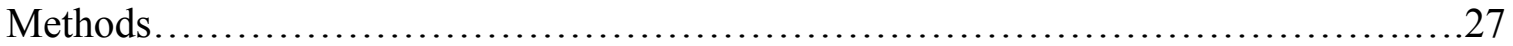

Results................................................................... 30

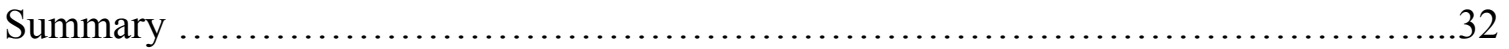

Conclusion and Limitations..................................................... 36

Recommendations and Implications for Advanced Practice.......................... 38

References..............................................................40

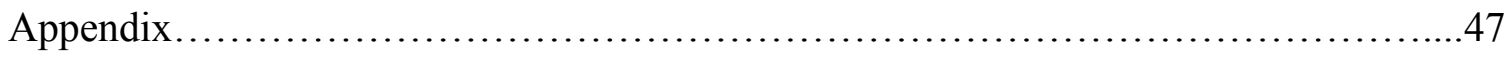


The Relationship Between Osteoarthritis, Hip Arthroplasty, And Post-operative Ambulation Distances

\section{Statement of the Problem}

Osteoarthritis (OA) is a disorder of the joints that is a common cause for disability in U.S. adults (Murphy \& Helmick, 2012). The pathophysiology of OA is multifactorial and complex. It is not initiated by an inflammatory reaction but inflammation results from joint damage; injury results in loss of cartilage in the joints. The body's compensatory mechanisms initiate bone remodeling and osteophyte formation. Swelling that results from damage to the joints is believed to intensify the long- term joint damage (Birchfield, 2001). Clinical features of OA include a creaking, crunching, and a grinding sensation on moving the joint, decreased range of motion, and pain with movement (Walker, 2011).

Osteoarthritis is strongly correlated with aging; the risk of OA increases considerably with each decade after the age of 45 years (Moskowitz, 2009). Risk factors for the disease include African American or Hispanic ethnicity, older age, female gender and hormonal status, genetics, bone density, nutrition, joint injury, obesity, occupation, physical activity, joint biomechanics, and muscle weakness (Garstang \& Stitik, 2006). The burden of suffering, pain and functional impairment, results in reduced quality of life, a primary concern of people with OA (Moskowitz, 2009).

Data from the National Health Interview Survey indicated that 52.5 million (22.7\%) of U.S. adults reported that their doctor had diagnosed them with arthritis; 22.7 million (9.8\%) of all U.S. adults have activity related limitations from their arthritis 
(Barbour et al., 2012). Data from Hootman and Helmick (2006) suggested that 67 million (25\%) U.S. adults over 18 years old will have a diagnosis of arthritis by the year 2030. Of the 67 million with an arthritis diagnosis by 2030, 25 million will also report activity limitations secondary to OA. In 2003, the estimated yearly total expenditure for OA was $\$ 128$ billion or $1 \%$ of the U.S. gross domestic product; $\$ 81$ billion in medical costs and $\$ 47$ billion in lost income (Murphy \& Helmick, 2012).

Recent legislation, the Affordable Care Act (ACA), intends to expand health insurance coverage, increase the insurance company's accountability, lower health care costs, guarantee more choices, and strengthen the quality of care for all enrolled Americans (Centers for Medicaid and Medicare Services, 2014). Stain, Hoyt, Hunter, Joyce, and Hiatt (2014), described the legislation's foci as, "High-value care (... that) emphasizes quality, safety, resource use and appropriateness, and the patient's experience of care" (p. 984). As the American baby boomer population ages and the obesity epidemic intensifies, the health care system will sustain a substantial financial impact in caring for these patients (Sun, Wu, and Kalunian, 2007). The increase in cost will put a strain on the already highly complex health care system recently restructured by the ACA.

According to Sun et al. (2007), the basis for OA treatment should be divided into three distinctly different categories: non-pharmacologic, pharmacologic, and surgical. Non-pharmacologic interventions include: weight loss, physical therapy, exercise programs, and braces and orthotics. Pharmacologic interventions include: treatment with analgesics (Tylenol, narcotics), nonsteroidal anti-inflammatory drugs (NSAIDs), steroid 
injections, viscosupplementation with hyaluronic acid (promotes connective tissue repair), arthroscopic irrigation, and nutraceuticals (over the counter supplements such as glucosamine and chondroitin which aid in connective tissue growth and repair). When the pharmacologic and non-pharmacologic approaches are not effective, the patient may seek surgical intervention to improve mobility and reduce pain, which increase the quality of life.

The purpose of the project was to quantify the ambulation distances of patients who have had total hip replacements (THR) using one of three surgical approaches. The timeframe for measurement was from the day of surgery (day 0 ) through the second postoperative day (POD 2) comparing different surgical approaches with post-operative ambulation distances.

Pre-operative educational classes, offered to elective joint replacement patients in the weeks prior to elective surgery, may play a significant role in post-operative recovery. After attending a pre-operative education class the patient's length of hospital stay was shorter, their mobilization was faster and functional scores improved faster, and the hospital also benefited financially (Moulton, Evans, Starks \& Smith, 2015). This provided the rationale for including pre-operative educational classes in this project.

The role of the nurse practitioner includes assuring the best patient outcomes in a cost-effective approach to patient care. The distance a patient ambulates may be a marker for readiness for discharge. Early discharge provides an economic benefit to the hospital and patient; the sooner a patient ambulates the requisite distance, he or she may be 
discharged earlier, reducing the overall economic burden of surgical treatment of OA to the health care system.

The purpose of this project was to measure the distance each patient ambulated and compare the three total hip arthroplasty approaches to investigate if a particular approach achieved better outcomes in terms of post-operative ambulation distance. If there were evidence from the results of this project to support a particular approach, the student researcher may share findings with the other members of the healthcare team. All members of the healthcare team strive to provide patient-centered, quality care in the most fiscally responsible manner. This project may contribute to achieving that outcome.

The literature review will provide findings that support the significance of osteoarthritis as a health problem for many individuals, discuss the therapeutic options, and describe the importance of mobilizing patients after surgery. The quantification of distance ambulated will be a marker for patients' achievement of mobility and be considered as evidence for timely discharge from the acute care setting. 


\section{Review of the Literature}

The student researcher, using Ovid SP and the Cumulative Index to Nursing and Allied Health Literature (CINAHL) databases, conducted a comprehensive search of the literature published between 1974 and 2015. Keywords used to conduct the search included: hip osteoarthritis, total hip replacement, total hip arthroplasty, hip surgical approach, hip lateral approach, hip posterior approach, hip anterior approach, total hip physiotherapy, post-operative mobility, total hip weight bearing, immobility, barriers to mobility, missed nursing care, and the Health Belief Model. The literature review encompassed the following topics: osteoarthritis, osteoarthritis treatments, surgical approach, early mobilization, post-operative mobility and physiotherapy, weight bearing exercises, and barriers to mobilization.

\section{Osteoarthritis}

Osteoarthritis (OA) is a degeneration of the synovial joints that results in loss of cartilage, changes in the underlying bone, and osteophyte formation. Disease characteristics include joint pain and stiffness, limited range of motion, and pain (Walker, 2009). Osteoarthritis is becoming more prevalent due to the aging population; it is a complex multifactorial disease with numerous risk factors (Merkle \& McDonald, 2009). Arden and Leyland (2013) described OA as a phenotype resulting from several different disease processes that affect the joints.

The causative agents of OA are obesity, reduced muscle strength, and joint morphology that result in erosive damage and pain (Arden \& Leyland, 2013). Obesity, which has both genetic and environmental influences, will ultimately increase the 
workload on the joint and promote an increased rate of joint degeneration. Reduced muscle strength, particularly quadriceps weakness, decreases joint stability thus reducing functional ability and increasing pain. Joint morphology (basic anatomical joint form and structure) is an inherited predisposition for OA, thus it is an important risk factor to assess (Arden \& Leyland, 2013). Erosive OA which results from joint injury and inflammation, particularly erosive hand $\mathrm{OA}$, has been found to require more pain medications to treat than non-erosive OA (Arden \& Leyland, 2013).

MacDonald, Sanmartin, Langlois, and Marshall (2014) conducted a study to estimate the time between the patient's symptom recognition of onset and physician's diagnosis of OA. A cross sectional survey of Canadian residents, the Survey on Living with Chronic Diseases in Canada, was conducted by the public health agency and used, in conjunction with the Canadian Community Health Survey, to screen and select a total of 1755 participants with OA. Respondents were asked about their experiences with joint pain, the time from symptom onset to physician diagnosis, pain medication use, and any additional treatments used such as physical therapy. Using descriptive statistics, the researchers found that $48.1 \%$ of patients were diagnosed with OA the same year their symptoms began, $41.6 \%$ had symptoms from one year to 7.7 years, ranging $6.4-8.9$ years before diagnosis, and $10.2 \%$ were diagnosed prior to symptom emergence. The average age of patients with an OA diagnosis was 50.4 years (confidence interval of 95\%). An estimated $39.4 \%$ of participants used prescription medications to treat OA (highest prevalence in the 65 and older age group) and $66.3 \%$ used non-prescription methods of treatment. The results of the study revealed that nearly half the patients (48.1\%) had an 
average of 7.7 years between symptom onset and diagnosis. It is important to emphasize that this is a key period in which to make lifestyle changes, with an emphasis on weight management (MacDonald et al., 2014).

\section{Osteoarthritis of the hip}

Osteoarthritis of the hip has been investigated as a specific site of pathology.

Sulsky et al. (2012) conducted a systematic review that examined the epidemiological evidence for an increased external workload as a causative factor in OA of the hip. The researchers screened 262 studies using exclusion criteria such as professional athletes, hip pain prior to occupation, and rheumatoid arthritis; six studies on workload or occupation and OA were included. They concluded that there was a consistent relationship between hip OA and heavy physical workload in scientific literature, but estimates on the magnitude of the effect and the specific occupational risks were not well described. There was consistent evidence for a causal association between heavy workload and risk for OA but nothing definitive that provided insight into specific high-risk occupations or situations. Differences in study design, lack of heterogeneity of participant occupations, and final selection of studies in English and German language proved to be limitations of this literature review.

Karrasch and Lynch (2014) described OA of the hip as having an insidious onset, which continues with symptoms that may wax and wane. Pain is most likely felt in the groin area and is relieved with rest. Risk factors include female gender, obesity, physical jobs, high-impact sports, high bone density, trauma, congenital or developmental abnormality, or a family history of OA. 
The pathophysiology of hip OA is complex. Sovani and Grogan (2013) described the onset of OA as marked by changes in the otherwise smooth and well-lubricated articular cartilage that lines the joint surfaces. Pathological changes include loss of the articular cartilage and changes in the boney structure by means of subchondral sclerosis and marginal osteophytes formation. A complex array of changes occur at the molecular level of cartilage resulting in the loss of tensile strength of the cartilage (which decreases its load bearing ability). Because cartilage lacks traditional blood supply, the cartilage cells respond to damaged cells by either performing modified tissue maintenance, remodeling, or by degrading the tissue. An imbalance between the tissue repair and tissue degradation will result in an imbalance and eventually cause OA in joints such as the hip.

OA is a complex disease that impacts the lives of patients. When found in the hip, OA can cause severely debilitating pain and limited function in patients. After a diagnosis is made through clinical presentation and radiographs, the next step is to develop a treatment plan.

\section{Osteoarthritis Treatment}

The goals of OA treatment are to reduce pain and stiffness, maintain or improve range of motion, and decrease the rate of joint damage. Non-invasive methods of treatment include pharmacological treatment (OTCs and prescribed analgesics) and nonpharmacological therapies such as muscle strengthening, the use of assistive devices, and weight loss. Invasive methods for treatment involve a referral to an orthopaedic surgeon 
and evaluation of options, including steroid injections to reduce joint inflammation and pain, and joint replacement surgery (Walker, 2009).

Grayson and Decker (2012) identified the failure to respond to non-invasive treatment and the presence of debilitating pain as the indications for a total hip arthroplasty. Total hip arthroplasty (total hip replacement, THR) is one of the most successful joint replacement surgeries according to Grayson and Decker (2012). It offers significant pain relief and in most cases it allows the patient to resume some low impact exercise and offers significant functional improvement. Complications include dislocation $(2 \%-3 \%)$, thromboembolism $(2 \%-3 \%)$, infection $(1 \%-2 \%)$, neurologic injury $(0.6 \%-3 \%)$, and vascular injury $(0.2 \%-0.3 \%)$. Data supports good survivability of most hip prostheses for more than 20 years post-operatively (Grayson \& Decker, 2012).

\section{THR Surgical Approaches}

Total hip replacement surgery involves excision of the bone ends and their replacement with artificial metal and plastic components, known as a hip prosthesis (Lucas, 2007). There are three surgical approaches to a hip replacement procedure:

posterolateral (posterior), lateral, and anterior (anterolateral). Each surgical approach has different indications, advantages, disadvantages, possible complications, and different post-operative care (Cheng, Feng, Liu, \& Zhang, 2009).

The first of three surgical approaches, the posterior approach, has been credited as the most common surgical approach. This approach provides adequate visualization of a hip joint and spares the abductor muscle from excessive trauma. The posterior approach is reported to have higher dislocation rates than the other two surgical approaches and has 
an increased risk of sciatic nerve injury (Petis, Howard, Lanting and Vasarhelyi, 2015). The posterior surgical approach requires post-operative, posterior hip movement precautions. These precautions limit hip flexion to 90 degrees or less (no bending at the waist), avoid excessive hip adduction (no crossing of legs), and limit excessive hip internal rotation (no twisting at the waist or pointing toes inward) (Enseki and Berliner, 2013).

The second surgical approach is the lateral approach. It gives adequate exposure of the bones (acetabulum and femur) for the surgeon to conduct the surgery. Proponents for this surgical approach described the benefits as a low dislocation rate and full visualization of the workspace by the surgeon. Opponents of the lateral surgical approach described post-operative insufficiency in the abductor muscles because they have been cut and manipulated during the surgery and the possibility for superior gluteal or femoral nerve injury (Petis et al. (2015). Patients undergoing lateral surgical approach of THR procedures do not have post-operative movement precautions (Enseki et al. 2013).

The third surgical approach is the anterior approach, which is gaining in surgeon preference (Petis et al., 2015). Advocates describe this approach as muscle sparing because it cuts through less muscle, gives early restoration of functional ability, and has a lower dislocation rate. Opponents describe the surgery as complicated by lack of direct visualization of the femur and acetabulum, and as having a risk of femoral nerve damage. Enseki et al. (2013) described this surgical approach as not consistently requiring postoperative movement precautions. Surgeon preference and individual cases dictate 
when these precautions are recommended; precautions would limit the motions of extension (bending leg backwards) and external rotation (pointing toes outward).

Each patient is a unique individual and must discuss with his/her surgeon which approach is best for his/her particular situation. A relevant question is, "Does the research show if a particular approach gives a patient any advantage in recovery?" The following is a review of literature comparing surgical approaches and associated complications.

\section{Comparison of Surgical Approaches}

Jolles \& Bogoch (2006), in a review of the literature, found 44 articles pertaining to total hip replacement and surgical approach. The 44 studies were reduced to four prospective cohort studies, which addressed their specific outcome measures. In these four studies, 241 patients were grouped according to the surgical approach. The reviewers found that the lateral approach may have slightly less initial post-operative improvements in range of motion, but overall there was no significant difference in overall functional improvements post-operatively as compared to the posterior approach. The researchers attributed the overall weakness in the studies to small patient sample size and general poor methodology. The reviewers found that the risk of dislocating the prosthesis and the chances of having difficulty ambulating post-operatively were the same with the posterior and lateral approaches. Jolles and Bogoch (2006) noted that the lateral approach had more potential to cause nerve damage than the posterior approach, but, given the small sample size, there was not enough evidence to support the finding with certainty. 
In a prospective observational study of 42,233 patients, Lindgren, Wretenberg, Karrholm, Garellick, and Rolfson (2014) reviewed the Swedish Hip Arthroplasty, a national registry of patients and found that the posterior approach demonstrated slightly better mean outcomes than the lateral approach. The researchers used the outcome measures of client reported post-operative satisfaction and pain relief as comparative factors. Of the 42,233 patients surveyed, 4,962 completed additional surveys through six post-operative years. After six years, the posterior approach provided better outcomes, indicating the long-term benefits of this surgical approach.

In a prospective, nonrandomized, multicenter study of 1,089 patients, researchers Palan, Beard, Murray, Andrew, and Nolan (2009), measured, for five years postoperatively, patient centered outcomes and post-operative complications. The researchers concluded that the posterior approach had better initial clinical outcomes including pain and functional levels through three months post-operative. In the long term, there was no difference in dislocation and revision rates through the five-year mark. A limitation of the study was the number of patients who completed the study: 43 patients were lost to follow up and 209 patients with incomplete results through five years, 163 of whom were incomplete in the first year. The researchers suggested that patient specific differences in anatomy, such as femoral head size, were more of an influence on dislocation complications than surgical approach.

In a prospective, comparative, nonrandomized study of 120 patients (60 anterior approach and 60 posterior approach), Rodriguez et al. (2014) found that the anterior surgical approach provided faster initial post-operative recovery. Functional levels for 
the anterior approach were significantly better for the initial two weeks, but there was no significant difference in the recovery after the six-week post-operative period.

A prospective randomized clinical trial (Barrett, Turner, \& Leopold, 2013) studied 87 patients with a focus on mobility status and pain level. The study evaluated the patients' ability to walk and climb stairs, in addition to their functional pain level at six weeks and three, six, and nine months post-operatively. The results noted significant initial benefits, for up to two weeks, with the anterior approach as compared to the traditional posterior or lateral approaches. After the six-week mark there were no differences among the surgical approaches.

Cheng et al. (2009) compiled twelve randomized or quasi-randomized studies with a total of 1,205 THR patients in a meta-analysis. It included both prospective and retrospective nonrandomized control studies and used only peer-reviewed papers. The researchers found the minimally invasive surgical approaches (anterior), had comparable long-term outcomes to traditional approaches (posterior and lateral). A significant difference in short-term outcomes, such as blood loss (decreased by an average of nearly $80 \mathrm{cc}$ over other approaches), was found in the minimally invasive total hip surgical approaches (anterior approach). There were no significant differences in overall operating time among the three surgical approaches (Cheng et al. 2009). Limitations to these studies were the small size of the comparative groups and the lack of long-term follow up in most of the groups.

Each of the three surgical approaches has both advantages and disadvantages. The surgical approach is simply a physician preference by training and experience; each 
surgical approach is safe and clinically efficacious. Further research in the debate of the THR surgical approach should focus on clinical outcomes, health care economics, and restoration of function (i.e., ambulation) post operatively (Petis et al., 2015).

\section{Post -Operative Mobility}

Kalisch, Lee, and Dabney (2014) reviewed thirty-six articles to evaluate physiological, psychosocial, social, and organizational outcomes of early mobilization of hospitalized adults. The strength of the study was the inclusion of patients, including post-operative patients, from different areas with a variety of illnesses and procedures. The researchers concluded that a benefit of mobilizing hospitalized adults was increased physical functioning levels, along with stimulating the patient's emotional and social well-being. The simple task of mobilization decreased patients' anxiety and stress while increasing their confidence, quality of life, independence, comfort, and satisfaction. In addition to these patient-centered benefits, early inpatient ambulation resulted in important organizational benefits, e.g. decreased length of stay, decreased mortality, and decreased cost (Kalisch et al., 2014).

Examining the effects of bed rest and immobility from a different perspective, Coker, Hays, Williams, Wolfe, and Evans (2014) investigated the changes in body composition, muscle strength, functional status, and the relationship between these factors in nineteen adults. The researchers found that short-term bed rest had many negative consequences including increased risk for deterioration in muscle strength, a decline in physical function, further reduction in muscle mass and aerobic exercise 
capacity, inability to perform activities of daily living, increased hospitalization, and an increase in morbidity and mortality (Coker et al., 2014).

In a prospective, observational cohort study, Brown, Redden, Flood, and Allman (2009) evaluated the amount of time older adult patients spent in the three stages of mobility: lying, sitting, and standing or walking. A survey of the patients' first four inpatient days found that the average participant spent approximately $83 \%$ of this time period lying down in bed. Unintended consequences of low mobility included functional decline, pressure ulcers, and increased fall risk (Brown et al., 2009).

Zisberg et al. (2011) conducted a study of 525 elderly patients, with nondisabling conditions, admitted to a large teaching hospital. The patients' mobility was assessed throughout their hospital course, at discharge, and at their one-month follow-up appointment. The researchers found that $45 \%$ of patients had decreased their activities of daily living at discharge, and the number increased to $49 \%$ at their follow-up appointment. There was a significant decline for $57 \%$ of participants in independent activities of daily living, such as cooking, washing clothes, and cleaning their homes, at their one-month follow-up. The researchers suggested that the more ill the patient, the less he or she moved following discharge. The conclusion was that decreased mobility is a modifiable risk factor and usually can be avoided to decrease functional decline (Zisberg et al., 2011).

\section{Perceived Barriers to Mobilization}

The below average activity levels of older adults in the hospital led Buttery and Martin (2009) to survey 66 hospitalized patients to assess their knowledge of the benefits 
of mobility. Of these 66 hospitalized patients, 44 completed their questionnaire prior to discharge. The surveyors found that the patients reported poor knowledge in areas of how mobility can positively affect chronic disease and that most patients believed their activity levels as adequate. Problems with their health, such as leg problems or breathing problems, were the most common reasons given for lack of activity. These actual or perceived barriers to mobilization have a negative effect on a patient's health, including the lack of exercise being detrimental to bone and joint health (Buttery and Martin, 2009).

In a prospective, observational cohort study $(n=45)$ done at a Veterans' Hospital by Brown et al. (2009), the researchers reported that patients often struggle with mobilization barriers. These barriers can be divided into three major groups: patient symptoms (pain, fatigue, and weakness), restraining medical devices (catheters and intravenous lines), and concern from patients and providers about falls. The patients also stated that the bed is the most comfortable and practical position to avoid the busy hospital hustle and bustle and that there is a lack of interesting places to go in these facilities. The researchers followed the mobility of the 45 elderly patient cohort while admitted to the hospital and found that, despite being able to walk prior to admission, these patients spent most of their day in bed (Brown et al., 2009).

Zisberg et al. (2011) conducted a study of 525 elderly patients, with nondisabling conditions, admitted to a large teaching hospital. The patients' mobility was assessed throughout their hospital course, at discharge, and at their one-month follow-up appointment. The researchers found that $45 \%$ of patients had decreased their activities of 
daily living at discharge, and the number increased to $49 \%$ at their follow-up appointment. There was a significant decline for $57 \%$ of participants in independent activities of daily living, such as cooking, washing clothes, and cleaning their homes, at their one-month follow-up. The researchers suggested that the more ill the patient, the less they moved following discharge. The conclusion was that decreased mobility is a modifiable risk factor and usually can be avoided to decrease functional decline (Zisberg et al., 2011).

Palmer (1995) described patients as motivated to get up and move around to avoid negative symptoms associated with bed rest, boredom, pain, and fatigue. Institutional barriers often prevent patients from maximizing their in-hospital mobility. Lack of staff and availability of assistive devices can often limit post-operative total hip patients from ambulating. Palmer (1995) reinforced the fact that mobility is a multidisciplinary problem and an interdisciplinary approach is needed to solve it. A patient centered collaborative approach to maximize post-operative mobility is key to overcoming mobility barriers and avoiding functional decline.

In a study examining mobility in older adults, Rantanen (2013) discussed the physiologic requirements for ambulation, the sensory factors and environmental barriers to ambulation. From a physiologic standpoint, a person must have adequate musculoskeletal, cardio-pulmonary, sensory and neural systems to provide the balance and strength in order to walk. Sensory factors, including vision and hearing loss, are significant barriers to mobility and often precipitate falls. Recent falls create a mental barrier, as the patient is afraid to fall again, thus creating another barrier and possibly 
escalating functional decline. Environmental barriers, such as lack of a safe indoor area for ambulation during winter weather, impact mobility for people with and without walking aids such as canes and walkers (Rantanen, 2013).

Through an eight step concept analysis, Kalisch, Landstrom, \& Hinshaw (2009) described the concept of 'missed nursing care' which applies to institutional constraints preventing patients from ambulating or receiving physiotherapy. Factors contributing to missed nursing care included: competing priorities in work load, available resources, 'systematic' elements of the nursing process and the internal perceptions, as well as, the habits and values of the nurse. Fear of repercussions, retribution or being blamed, and unstandardized reporting of the problem were possible reasons for nursing care being missed. The consequences of missed nursing care have both patient-centered and institutional implications that can prove costly to patients and the health care system (Kalisch et al., 2009).

\section{Post-Operative Physiotherapy of THR patients}

Post THR physiotherapy exercises need to be a concise group of well chosen exercises that are easy for the patient to follow and are initiated early in the recovery process (Bandholm \& Kehlet, 2012). In a randomized control trial of 100 patients by Monticone et al. (2014), 40 men and 60 women were divided into two research cohorts: one with early and full weight bearing and one with partial weight bearing and traditional kinetic exercises (range of motion and strengthening exercises performed while lying in bed). The results indicated that the early physiotherapy group had better functional and quality of life survey scores than the other group. Based on these findings, the 
researchers recommended personalized task-oriented rehabilitation early in the recovery process to improve functional outcomes and hip range of motion.

Davis (2012) conducted a meta-analysis, using physiotherapy participation and performance as the outcome measures, including eight studies that focused on the rehabilitation of patients with OA of the hips and knees. The meta-analysis revealed the necessity for patient-centered outcome goals, which reinforced the need for individualizing physiotherapy. A key element was that measures of participation were feasible and measurable goals, which may encourage mobilization of total hip replacement patients post-operatively.

Through a retrospective chart review and follow-up surveys of 658 THR patients over a two year period, at a private pay institution, Ieiri et al. (2013) measured therapeutic efficiency by giving patients a set plan of post operative exercises, advancing as they progress post-operatively, and following the patients through their second post-operative follow-up appointment. Outcome measures gathered at follow-up included age, sex, body mass index, complications, living alone, contralateral hip OA, range of hip joint motion, walking aids, and pre-operative mental health. The researchers noted that intensive post-operative therapy is particularly necessary for total hip replacements, especially ones with contralateral hip OA, severely restricted range of motion in the operative hip, and those who used a walking aid prior to surgery.

The benefits of post-operative physiotherapy are well documented in the literature as being beneficial to improve joint range of motion and promote increased functional 
status. A concern is to determine the timeline for safe and beneficial initiation of physiotherapy post-THR.

\section{Early Mobilization of THR patients}

According to the American Academy of Orthopedic Surgeons (AAOS) (2007, 2011), light activity, such as walking, is key to post-operative THR recovery and should begin within the first twenty-four hours post-operatively. Early post-operative exercises are important for increasing circulation to the lower extremities to prevent blood clots, to strengthen muscles, and to improve hip movement. Barker et al. (2013) stated that early post-operative improvement of hip motion is safe and practical for total hip replacement patients as early as the day of surgery.

In a randomized control trial of 100 patients at a rehabilitation facility, Monticone et al. (2014) compared groups of post THR patients who underwent traditional kinetic exercise with those who participated in a task-oriented exercise plan. The traditional kinetic exercise program was aimed at improving joint range of motion through repetition. These exercises included hip flexion/extension, abduction, controlled external rotation, strengthening of the quadriceps and walking. All of these exercises were performed while lying in bed. The task-oriented exercise program included functions that were targeted at improving functional status and the patient's activities of daily living. These exercises included moving from a sitting to standing position, ascending and descending stairs, starting and stopping, balance, obstacles, walking and/or cycling. Using a tool developed by the Western Ontario and McMaster Universities, the Western Ontario and McMaster Osteoarthritis Index found that the post-operative task-oriented 
exercise showed a 22 point decrease in physical function scores compared to only a 9.4 point reduction in the kinetic exercise group. These results were a positive indicator of increased postoperative function in those patients who engaged in kinetic exercise. Regardless of group, the researchers stated that exercise should speed recovery, diminish disability quicker, and ultimately reduce postoperative pain (Monticone et al., 2014).

In response to two meta-analyses indicating the inadequacies of post-operative hip physiotherapy, Bandholm \& Kehelt (2012) suggested that early physiotherapy, which includes strength training, should be instituted as soon as medically allowed in the postoperative recovery period of a total hip replacement. . The researchers concluded that early physiotherapy can decrease hospitalization length, enhance recovery, and decrease risk for medical complications, but it must be tailored to the patient's specific situation. There is a need for more intensive, simple, and straight-forward physiotherapy from the initial post-operative consultation through the recovery course (Bandholm \& Kehelt, 2012).

Larsen, Hansen, \& Soballe (2008) designed a study $(\mathrm{n}=98)$ of two post-operative THR groups to compare normal physiotherapy with fast track physiotherapy outcomes. Mobility, usual activities, pain, self-care, and anxiety were used as measurement tools at the patients' three-month follow-up appointment. The researchers found that the fast track physiotherapy patients had $10 \%$ higher scores on their three-month follow-up surveys as compared to standard physiotherapy. Larsen et al. (2008) noted that the shorter hospital stay and earlier mobilization directly correlated to a reduction in deterioration and quicker regain of physical performance. 


\section{Full Weight Bearing Exercise}

Barker et al. (2013) conducted a randomized control trial of eighty males in an orthopedic specialty hospital to investigate a tailored rehabilitation program for patients who underwent a total hip replacement. The study group was divided into two cohorts that received full weight-bearing exercise either traditionally or by individualized physiotherapy. The results were measured by the criteria of functional scores, range of motion, strength, and patient goals. At one-year post-operative, $80 \%$ of the intervention group with the tailored rehabilitation met their goals as compared to 55\% of the standard physiotherapy control group. The researchers concluded that individualized full weight bearing from day one has been shown to be effective in improving functional outcomes and hip range of motion in patients undergoing THR (Barker et al., 2013).

Through a prospective randomized study, Thien, Karrholm, Ahnfelt, Stromberg, and Eriksson (2007) recruited forty-three patients who underwent an uncemented total hip arthroplasty (prosthesis relies on normal joint pressure and stress to create bony ingrowth, thus securing the hip instead of using cement). The patients were then assigned to either a partial weight bearing or full weight bearing cohort. Thien et al. (2007) measured possible migration of the hardware in both cohorts to investigate the implications for different post-operative weight bearing orders. Researchers noted that there was no difference in the groups at 3,6, and 52-week follow-up appointments. These findings are consistent with a previous study (Barker et al., 2013) that supported full weight bearing to the extent a patient can tolerate post-operatively. Full-weight bearing 
status is appropriate for uncemented total hip replacements and does not appear to affect the implanted prosthesis (Thien et al., 2007).

Husby et al. (2009) performed a randomized controlled study of 24 THR patients in a rehabilitation setting. The patients were divided into two equal groups, one that underwent maximal (full-weight bearing) strength training post THR and the other group underwent conventional (partial-weight bearing) rehabilitation post THR. Outcomes were measured by leg press repetitions, abduction strength, rate of force development, work efficiency, gait pattern, and quality of life. The maximal group out performed the conventional group in leg press $(p<0.002)$, abduction $(p=0.002)$, force $(p=0.030)$ and work efficiency $(p=0.065)$. Husby et al. (2009) concluded that maximal strength training was an efficient treatment to regain muscle strength post THR.

Osteoarthritis is a common and disabling disease that should be initially treated through conservative measures. When conservative measures fail, a THR may be indicated. THR can be done by one of three major surgical approaches: posterior, lateral, or anterior. Each of these surgical approaches has individual risks and benefits and is usually selected according to the surgeon training and expertise. The importance of mobility, along with perceived barriers to mobility is well documented in the research. Individualized full weight bearing physiotherapy, as soon as clinically advised for patients undergoing a THR, is well supported in the literature.

The project will use the Health Belief Model to help explain patients' motivation for change and their willingness to cooperate in post-operative ambulation as a means to 
improve their post-operative recovery. Discussion of this model is included in the next section. 


\section{Theoretical Framework}

The Health Belief Model (HBM), according to Rosenstock (1974), was developed by a group of social psychologists, working for the United States Public Health Service, who sought to improve the public's use of preventative services. The HBM explains health behaviors in terms of several constructs: perceived susceptibility, perceived severity of problem, perceived benefit of change, perceived barriers to action, and cues to action (Rosenstock, 1990).

Perceived susceptibility is subject to a person's perception of the risk of contracting a condition. This idea, along with the person's feelings and knowledge regarding the perceived severity of the disease or illness, will create a personal perceived threat for the individual. Acceptance of the perceived threat will ultimately lead an individual to consider what he/she believes to be the perceived benefits to a specific health change. Once benefits are identified, the patient's perceptions of barriers are considered. A cue to action then takes place; the person is able to adequately overcome his/her barriers, understand his/her susceptibility, comprehend the severity of the problem to see the benefit, and ultimately make the change (Janz \& Becker, 1984).

Pre-operative THR patients have already undergone the five constructs of the HBM and agreed to have surgery. The patients have perceived their own susceptibility to OA, perceived severity of the OA in terms of pain and functional limitations, perceived benefit of a total hip replacement, considered the barriers to action, and are cued to action. Once surgery is performed, the patients must start at the first construct, by this time their focus is on recovery. In this project, the patients must perceive their 
susceptibility to and severity of post-operative complications of immobility, including thromboembolism, pneumonia, and joint contractures. Postoperative patients must perceive a benefit to ambulation, not only decreasing complications but increasing joint range of motion, return of functional ability and quality of life. The nursing staff must work with the patients to determine their perceived barriers and subsequently provide education and encouragement to cue the newly post-operative patients to action to maximize post-operative mobility.

Using the Health Belief Model for guidance, the student researcher designed a research project to investigate the post-operative ambulation distances of a sample of THR patients in the immediate post-operative period. The methods of this project are presented in the next section of this paper. 


\section{Methods}

The purpose of this project was to quantify the ambulation distances of patients who had one of the three approaches to a THR. The timeframe for measurement was from the day of surgery (day 0) through the second post-operative day (POD 2) and, corresponding with different surgical approaches, compare the distances. Expedited approval from the Lifespan IRB and exemption from further review by the Rhode Island College IRB were obtained. The student researcher completed a retrospective chart review on an orthopedic nursing unit in a small community teaching hospital, collecting IRB approved data on 20 THR patients for each surgical approach.

\section{Procedures}

Expedited approval from the Lifespan IRB and exemption from further review by the Rhode Island College IRB were obtained. To gain access to the electronic medical record (EMR), the student researcher sought support from the Total Joint Center (TJC) Coordinator, who has organizational responsibility for the security of the Medical Record Numbers (MRN) and patient EMRs. The student researcher discussed the project objectives with the TJC coordinator and requested access to the MRN of the THR patients for a specific time frame. The TJC coordinator granted access and the student researcher obtained a list of MRN using a DRG code. The student researcher reviewed EMR of patients who had a THR between 06/01/14 and 12/31/14. Electronic medical records were reviewed to obtain a sample of 20 patients corresponding with each total hip surgical approach (anterior, posterior, and lateral). Data were recorded on an electronic 
spreadsheet (Appendix A), kept confidential, and stored de-identified in an encrypted drive to which only the researcher had access.

\section{Measurements}

The tool for collecting and inputting data on the electronic spread sheet was developed to guide the researcher to exclude the patients who did not have the diagnosis of OA and those patients who had a previous total hip replacement. The tool was developed to collect basic demographic information and document attendance for THR preoperative education class on for each of the surgical intervention group. The surgical approach and post-operative ambulation distances were retrieved corresponding with this project's stated purpose.

Data points collected were previous total hip procedure, diagnosis of OA, age, gender, weight, height, pre-operative education class attendance, surgical approach, and ambulation distances on post-operative day zero through two. Ambulation distances were consistently charted in the physical therapy notes and more infrequently in nursing notes in units of feet. Specific framed pictures on the hallway walls of the orthopedic unit were designed to be approximately 15 feet apart so that staff could measure ambulation distances consistently. The orthopedic unit standard for measuring ambulation included a measured distance of three feet from bed to bedside chair and a distance of ten feet from the bed to the bathroom or the doorway.

\section{Data Analysis}

Descriptive statistics including ranges and averages of selected data points were calculated, using a formula on the electronic spread sheet, to describe characteristics of 
the study's sample of THR patients. Average patient BMI and age were calculated for each surgical approach (anterior, posterior, and lateral). The gender composition of each surgical approach (anterior, posterior, and lateral) was totaled to describe the surgical cohorts. The attendance of a pre-operative education class, or the lack of attendance of this class, was considered to further describe the surgical groups. The average ambulation distances, POD 0-2 were tabulated and graphed by surgical approach. 


\section{The Results}

A total of approximately 80 charts were reviewed to obtain the desired 20 patients from each of the anterior, posterior, and lateral surgical approaches between 06/01/2014 and $12 / 31 / 2014$. Patients were excluded if they did not have a diagnosis of OA and if they had a previous THR. For the complete sample of 60 patients, the overall age range was 44-88 years old with a BMI range of 20.01-44.11. There were 4 males and 16 females in the anterior group, 12 males and 8 females in the posterior group and 11 males and 9 females in the lateral group.

To describe the demographics of each surgical approach group, the student researcher computed the average age and BMI. The anterior approach had an average age of 65.15 years, ranging from 49-85 years old and average BMI of 31.14, ranging from 23.96-44.11. The posterior approach had an average age of 61.40 years, ranging from 44-82 years old and average BMI of 31.28, ranging from 21.95-41.96. The lateral approach had an average age of 66.00 years, ranging from 62-80 years and average BMI of 29.71, ranging from 20.01-38.09.

Overall, 5 patients ( $8 \%$ ) of the 60 total did not attend a preoperative education class. The surgical approaches of these 5 patients are as follows; 3 posterior, 1 lateral, and 1 anterior. The average age for these 5 patients was 58.4 years old with an average BMI of 25.6 .

Table 1 shows surgical approaches as related to average ambulation distances. Each of the surgical approaches was compared, in terms of distance ambulated, from POD 0 , through each of two therapy sessions (\#1 and \#2) on POD 1 and 2. The posterior 
approach averaged $11.75 \mathrm{ft}$ on POD 0. The anterior approach averaged $60.80 \mathrm{ft}$ on POD 1,\#1. The posterior approach averaged $79.55 \mathrm{ft}$ on POD 1, \#2. The lateral approach averaged 92.22ft on POD2, \#1 and 93.33ft on POD 2, \#2.

Table 1 Average post-operative ambulation distances by surgical approach on POD 0, POD 1 and 2 each with physical therapy sessions (\#1 and \#2).

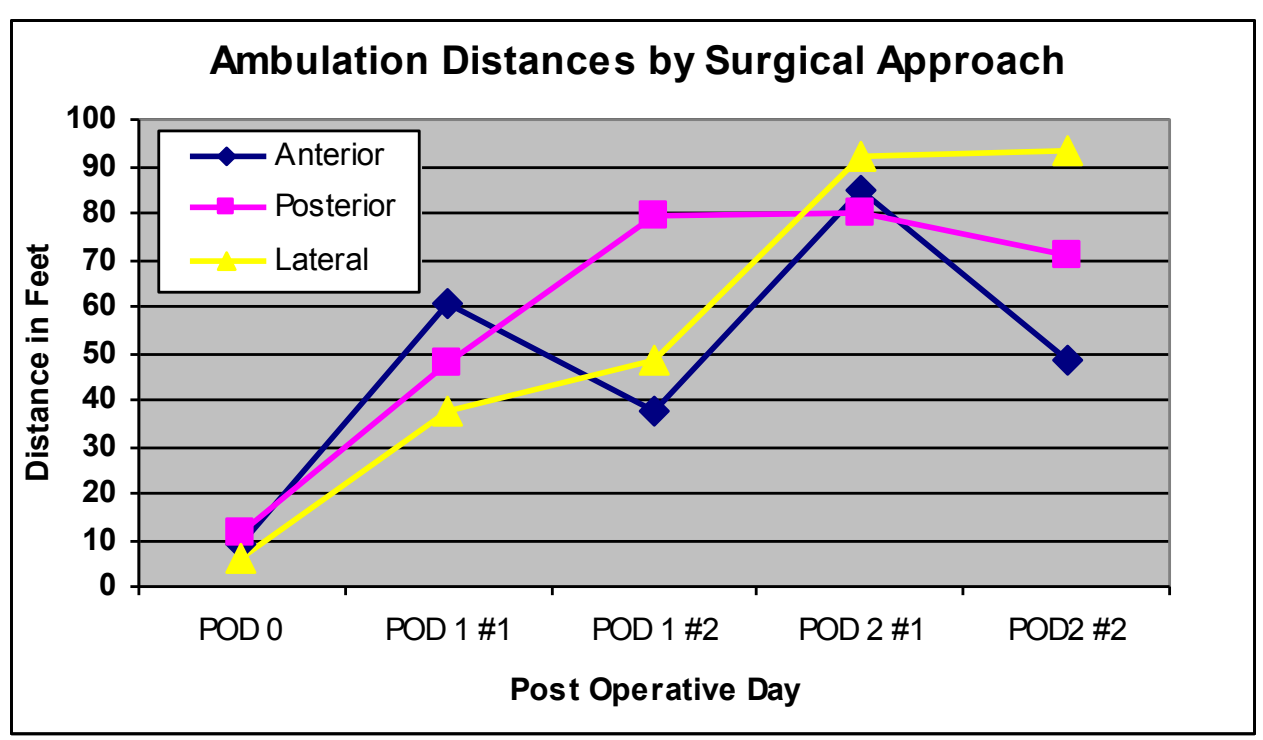




\section{Summary}

The purpose of this project was to measure the distance each patient ambulated and relate to each of three total hip arthroplasty approaches to investigate if a particular approach achieved better outcomes in terms of ambulation distance. The literature supported early full weight bearing ambulation for postoperative total hip replacement patients. However, the advantages and disadvantages for each total hip replacement surgical approach postoperatively were disputed and unclear. A retrospective chart review was completed using 20 patients from each total hip surgical approach (anterior, posterior, and lateral). Using a DRG code, a list of MRN was obtained and the data recorded on an electronic spreadsheet. The data were then tabulated, averaged, and the results compared to each other.

To describe the characteristics of the samples obtained, the student researcher recorded the gender, age, BMI, and attendance at a pre-operative education class. Each of these four characteristics of the sample will be discussed with surgical approach first, and then a comparison of each of the surgical approach cohorts results related to ambulation distances.

\section{Gender}

Two of the three surgical approach cohorts were distributed evenly. There were 12 males and 8 females in the posterior approach, and 11 males and 9 females in the lateral approach. The anterior approach favored females in this project; there were 16 females and 4 males. 


\section{Ages}

Two of the average ages of the surgical approach cohorts were found to be similar. The anterior approach had an average age of approximately 65 years (range 49$84 \mathrm{yrs}$ ) and the lateral approach had an average age of 66 years (range 49-86yrs). The posterior approach did have a slightly lower average age at approximately 61 years (range 44-88yrs). Although the posterior approach did have a lower average age, all three surgical approaches had a very similar age range from patients in their 40's all the way through patients in their 80 's.

\section{BMI}

BMI was described to illustrate the cohort characteristics and to assure that one surgical approach did not have an advantage over another by having a markedly lower BMI. The approximate results were: lateral, 29.71, the posterior, 31.28 and the anterior, 31.95. The findings suggest that all three surgical approaches were relatively close in BMI, which would not seem to give one cohort an advantage over another. The Harris Hip Score (HHS) is a tool commonly used to evaluate pain, limp, support, distance walked, stairs, ability to put on socks and shoes, sitting, deformity and range of motion in postoperative total hip replacement patients. According to the work of Yeung et al. (2011), the overall HHS scores of post-operative THR patients were significantly lower in obese patients (indicating less improvement) than the non-obese patients (indicating more improvement) in each category except for pain. This is an indication that nonobese patients progressed towards normal functioning faster than the obese patients and therefore would have affected the outcomes of this study. 


\section{Pre-operative Education Class}

Overall there were only 5 patients that did not attend the pre-operative education class; 3 were posterior approach, 1 was lateral approach and 1 was anterior approach. The average age for these 5 patients was 58.4 years old with an average BMI of 25.6, indicating this group of patients was slightly younger than and had a slightly smaller BMI than the general groups of surgical approach patients (anterior average age: 65.15 year with average BMI 31.95; posterior average age: 61.4 years with average BMI 31.28; lateral average age: 66 years and average BMI 29.71).

\section{Ambulation Distances by Surgical Approach}

Table 2 summarizes the ambulation distances by surgical approach and highlights in bold, signifies the greatest ambulation distance for that particular POD.

Table 2

Ambulation distances by surgical approach.

\begin{tabular}{|l|c|c|c|c|c|}
\hline & POD 0 & POD 1 \#1 & POD 1 \#2 & POD 2 \#1 & POD 2 \#2 \\
\hline Anterior & 8.9 & $\mathbf{6 0 . 8}$ & 37.86 & 85.05 & 48.57 \\
\hline Posterior & $\mathbf{1 1 . 7 5}$ & 47.80 & $\mathbf{7 9 . 5 5}$ & 80.00 & 70.71 \\
\hline Lateral & 6.30 & 37.40 & 48.25 & $\mathbf{9 2 . 2 2}$ & $\mathbf{9 3 . 3 3}$ \\
\hline
\end{tabular}

The anterior approach is traditionally credited with having patients up and moving more quickly post-operatively. According to this project's results, the posterior approach group on average ambulated the farthest on POD 0 . The anterior group ambulated $60.8 \mathrm{ft}$ on average, 12-22ft more than the other surgical approaches on POD 1 therapy session \#1. This then changed on the POD 1 therapy session \#2 when posterior approach 
ambulated the farthest with nearly an average of $80 \mathrm{ft}$. It is important to mention here that, if the patient met the goals set by the physical therapy team during the first physical therapy session of the day, then the patient did not receive a second session from the physical therapist. Instead the ambulation was supervised by the nursing staff, who did not document ambulation distances as reliably (if at all) in their notes. Six out of 20 patients of the anterior approach met their goals and did not have an ambulation distance documented for POD 1 therapy session \#2. The posterior and lateral approaches did not lose any patients to this, which indicated a greater number of anterior approach patients were making their physical therapy goals immediately on POD 1 therapy session \#1. The second POD, the lateral approach ambulation distances averaged over $12 \mathrm{ft}$ more than the other two surgical approaches during POD 2 therapy session \#1 and \#2. On POD 2, 37 out of 60 patients were discharged (among all 3 surgical approaches) before their second therapy session, making the sample size small and the results for POD 2 therapy session \#2 unreliable. 


\section{Conclusion}

The anterior surgical approach favored females in this research study, but age and BMI demonstrated no differences between the surgical approaches. Pre-operative education classes were found to be beneficial suggesting that they promote ambulation sooner after surgery than the patient who did not attend the class, but small sample size of the group who lacked the education class may have limited these results.

The ambulation distances between the three surgical approaches did not favor any particular surgical approach over another. The posterior approach had the farthest ambulation distance on the day of surgery. The following morning on POD 1 therapy session \#1, the anterior approach had the best ambulation distance. POD 1 therapy session \#2 data were not reliable because 6 of 20 patients in the anterior approach reached their therapy goals and did not get a second therapy session. POD 2 therapy session \#1 showed an advantage in the lateral approach, but POD 2 therapy session \#2 is highly limited since 37 of 60 patients were discharged from the hospital before the POD 2 therapy session \#2.

\section{Limitations}

Limitations to the ambulation distances were, once patients made their goals for physical therapy during the morning ambulation, they did not receive a second physical therapy session from the physical therapists that day. Instead the nursing staff assisted the patients to ambulate and the documentation for this distance was either not charted or not clearly measured. This further reduced an already small sample size. Also, the 
researcher did not account for the patients who either refused therapy during any of their session (related to pain or post-operative nausea) or could only perform kinetic exercises in bed. These patients received a $0 \mathrm{ft}$ ambulation distance in therapy documentation, which contributed to a smaller average for that surgical approach's ambulation distance. If patients were discharged from the hospital, they were not counted in the averages, thus further reducing a sample size. Although an important feature of research, the researcher did not attempt to gather any data regarding culture or ethnicity. This could be included in future studies to account for differences related to cultural or ethnicity factors. 


\section{Recommendations and Implications for Advanced Practice}

This research project found no clear-cut advantages in post-operative ambulation distances by surgical approaches. In the advanced practice role, the nurse practitioner would need to work with the staff nurses to standardize the documentation of ambulation distances and to teach patients about the importance of post-operative ambulation. Accurate charting on each patient and less patients refusing physical therapy may allow for clearer results on a research project similar to this one. Further implications for research may be to expand the sample size of this study and to exclude patients who either refuse or are medically unable to participate in physical therapy. Using more than one institution for the patient sample may aid in the validity of a similar research project.

Currently, total hip replacement surgical approaches are subject to the patient's choice of surgeon and his/her expertise. It is unclear if any particular surgical approach provides significant advantage in post-operative ambulation distances, a fact that is already widely supported in the literature. Interdisciplinary practice between the APRN, the staff RN, the surgeon, and the physical therapy staff varies greatly. Patient centered care needs to remain the focus of these surgeries while still encouraging the patient to reach an improved post-operative functional level upon discharge.

With billions of U.S. dollars being spent on osteoarthritis treatment, some of that being total hip replacements, and expanded health care coverage because of the Affordable Care Act, all members of the health care team must be fiscally minded. Continued research on which total hip surgical approach provides an advantage postoperatively while still giving patient-centered care may relate to improved patient 
outcomes, decreased length of hospitalization, and ultimately decrease the financial strain on the already highly complex health care system.

Technology, such as a pedometer worn on the wrist, may be a feasible solution to allow for correct documentation and accurate measurement of post-operative steps. This device can be used in the hospital and continue to be used after discharge to motivate the patient to ambulate and help patients quantify and document their activity. Having a physical marker for a post-operative recovery course over a period of months may provide incentive for activity and decrease the time a patient needs to fully recover. Decreasing the time to fully recover and return to pre-operative activities (i.e.: employment), will improve the population health and may decrease the financial strain on the health care system and contributing to safe, high quality, patient-centered care. 


\section{References}

American Academy of Orthopedic Surgeons. (2007, July). Total hip replacement exercise guide. Retrieved from http://orthoinfo.aaos.org/topic.cfm?topic=A00303

American Academy of Orthopedic Surgeons. (2011, December). Total hip replacements. Retrieved from http://orthoinfo.aaos.org/topic.cfm?topic=A00377

Arden, N. K., \& Leyland, K. M. (2013). Osteoarthritis year 2013 in review: Clinical. Osteoarthritis and Cartilage, 21(10), 1409-1413.

Bandholm, T., \& Kehlet, H. (2012). Physiotherapy exercise after fast-track total hip and knee arthroplasty: Time for reconsideration? Archives of Physical Medicine and Rehabilitation, 93(7), 1292-1294.

Barbour KE, Helmick CG, Theis KA, Murphy LB, Hootman JM, Brady TJ, Cheng YJ. (2012). Prevalence of doctor-diagnosed arthritis and arthritis-attributable activity limitation-United States, 2010-2012. MMWR 2013, 62(44), 869-873.

Barker, K. L., Newman, M. A., Hughes, T., Sackley, C., Pandit, H., Kiran, A., \& Murray, D. W. (2013). Recovery of function following hip resurfacing arthroplasty: A randomized controlled trial comparing an accelerated versus standard physiotherapy rehabilitation programme. Clinical Rehabilitation, 27(9), 771-784.

Barrett, W. P., Turner, S. E., \& Leopold, J. P. (2013). Prospective randomized study of direct anterior vs postero-lateral approach for total hip arthroplasty. The Journal of Arthroplasty, 28(9), 1634-1638.

Birchfield, P. C. (2001). Osteoarthritis overview. Geriatric Nursing, 22(3), 124-131. 
Brown, C. J., Redden, D. T., Flood, K. L., \& Allman, R. M. (2009). The underrecognized epidemic of low mobility during hospitalization of older adults. Journal of the American Geriatrics Society, 57(9), 1660-1665.

Buttery, A. K., \& Martin, F. C. (2009). Knowledge, attitudes and intentions about participation in physical activity of older post-acute hospital inpatients. Physiotherapy, 95(3), 192-198.

Centers for Medicaid and Medicare Services. (2014). Affordable care act. Retrieved from http://www.medicaid.gov/AffordableCareAct/Affordable-Care-Act.html

Cheng, T., Feng, J. G., Liu, T., \& Zhang, X. L. (2009). Minimally invasive total hip arthroplasty: A systematic review. International Orthopaedics, 33(6), 1473-1481.

Coker, R. H., Hays, N. P., Williams, R. H., Wolfe, R. R., \& Evans, W. J. (2014). Bed rest promotes reductions in walking speed, functional parameters, and aerobic fitness in older, healthy adults. The Journals of Gerontology Series A: Biological Sciences and Medical Sciences, glu123.

Davis, A. M. (2012). Osteoarthritis year 2011 in review: Rehabilitation and outcomes. Osteoarthritis and Cartilage, 20(3), 201-206.

Enseki, K. R., \& Berliner, M. (2013). Rehabilitation Following Total Hip Arthroplasty Surgery. Topics in Geriatric Rehabilitation, 29(4), 260-267.

Garstang, S. V., \& Stitik, T. P. (2006). Osteoarthritis: Epidemiology, risk factors, and pathophysiology. American Journal of Physical Medicine \& Rehabilitation, 85(11), S2-S11. 
Grayson, C. W., \& Decker, R. C. (2012). Total joint arthroplasty for persons with osteoarthritis. $P M \& R, 4(5)$, S97-S103.

Hootman JM, Helmick CG. (2006). Projections of U.S. prevalence of arthritis and associated activity limitations. Arthritis Rheum, 54(1), 266-229.

Husby, V. S., Helgerud, J., Bjorgen, S., Husby, O. S., Benum, P., \& Hoff, J. (2009). Early maximal strength training is an efficient treatment for patients operated with total hip arthroplasty. Archives of Physical Medicine and Rehabilitation, 90(10), 16581667.

Ieiri, A., Tushima, E., Ishida, K., Abe, S., Inoue, M., \& Masuda, T. (2013). What predicts 36-item health survey version 2 after total hip arthroplasty. Archives of Physical Medicine and Rehabilitation, 94(5), 902-909.

Janz, N. K., \& Becker, M. H. (1984). The health belief model: A decade later. Health Education \& Behavior, 11(1), 1-47.

Jolles, B. M., \& Bogoch, E. R. (2006). Posterior versus lateral surgical approach for total hip arthroplasty in adults with osteoarthritis. Cochrane Database of Systematic Reviews, 3(3).

Kalisch, B. J., Lee, S., \& Dabney, B. W. (2014). Outcomes of inpatient mobilization: A literature review. Journal of Clinical Nursing, 23(11-12), 1486-1501.

Kalisch, B. J., Landstrom, G. L., \& Hinshaw, A. S. (2009). Missed nursing care: A concept analysis. Journal of advanced nursing, 65(7), 1509-1517.

Karrasch, C., \& Lynch, S. (2014). Practical approach to hip pain. Medical Clinics of North America, 98(4), 737-754. 
Larsen, K., Hansen, T. B., \& Soballe, K. (2008). Hip arthroplasty patients benefit from accelerated perioperative care and rehabilitation: A quasi-experimental study of 98 patients. Acta Orthopaedica, 79(5), 624-630.

Lindgren, J., Wretenberg, P., Kärrholm, J., Garellick, G., \& Rolfson, O. (2014). Patientreported outcome is influenced by surgical approach in total hip replacement: A study of the Swedish Hip Arthroplasty Register including 42233 patients. Bone \& Joint Journal, 96-B(5), 590-596. doi:10.1302/0301-620X.96B5.32341

Lucas, B. (2007). Preparing patients for hip and knee replacement surgery. Nursing Standard, 22(2), 50-56.

MacDonald, K. V., Sanmartin, C., Langlois, K., \& Marshall, D. A. (2014). Symptom onset, diagnosis and management of osteoarthritis. Health reports, 25(9), 10-17.

Merkle, D., \& McDonald, D. D. (2009). Use of recommended osteoarthritis pain treatment by older adults. Journal of advanced nursing, 65(4), 828-835.

Monticone, M., Ambrosini, E., Rocca, B., Lorenzon, C., Ferrante, S., \& Zatti, G. (2014). Task-oriented exercises and early full weight-bearing contribute to improving disability after total hip replacement: A randomized controlled trial. Clinical Rehabilitation, 28(7), 658-668.

Moulton, L. S., Evans, P. A., Starks, I., \& Smith, T. (2015). Pre-operative education prior to elective hip arthroplasty surgery improves postoperative outcome. International Orthopaedics, 1-4.

Moskowitz, R. W. (2009). The burden of osteoarthritis: Clinical and quality-of-life issues. The American Journal of Managed Care, 15 (8 Suppl), S223-9. 
Murphy, L., \& Helmick, C. G. (2012). The impact of osteoarthritis in the United States: A population-health perspective. The American Journal of Nursing, 112(3), S13S19.

Palan, J., Beard, D. J., Murray, D. W., Andrew, J. G., \& Nolan, J. (2009). Which approach for total hip arthroplasty: anterolateral or posterior? Clinical Orthopaedics and Related Research, 467(2), 473-477.

Palmer, R. M. (1995). Acute hospital care of the elderly: Minimizing the risk of functional decline. Cleveland Clinic Journal of Medicine, 62(2), 117-128.

Petis, S., Howard, J.L., Lanting, B. L. \& Vasarhelyi, E.M. (2015). Surgical approach in primary total hip arthroplasty: Anatomy, technique and clinical outcomes. Canadian Journal of Surgery, 58(2), 128-139.

Rantanen, T. (2013). Promoting mobility in older people. Journal of Preventive Medicine and Public Health, 46(Suppl 1), S50-S54.

Rodriguez, J. A., Deshmukh, A. J., Rathod, P. A., Greiz, M. L., Deshmane, P. P., Hepinstall, M. S., \& Ranawat, A. S. (2014). Does the direct anterior approach in THA offer faster rehabilitation and comparable safety to the posterior approach? Clinical Orthopaedics and Related Research, 472(2), 455-463.

Rosenstock, I. M. (1974). Historical origins of the health belief model. Health Education \& Behavior, 2(4), 328-335.

Rosenstock, I. M. (1990). The health belief model: Explaining health behavior through expectancies. 
Sovani, S. \& Grogan, S. (2013). Osteoarthritis detection, pathophysiology and current/future treatment strategies. Orthopaedic Nursing, 23(1), 25-36.

Stain, S. C., Hoyt, D. B., Hunter, J. G., Joyce, G., \& Hiatt, J. R. (2014). American surgery and the Affordable Care Act. JAMA Surgery, 149(9), 984-985.

Sulsky, S. I., Carlton, L., Bochmann, F., Ellegast, R., Glitsch, U., Hartmann, B., Glitsch, U. \& Sun, Y. (2012). Epidemiological evidence for work load as a risk factor for osteoarthritis of the hip: a systematic review. PloS One, 7(2), e31521.

Sun, B. H., Wu, C. W., \& Kalunian, K. C. (2007). New developments in osteoarthritis. Rheumatic Disease Clinics of North America, 33(1), 135-148.

Thien, T. M., Karrholm, J., Ahnfelt, L., Stromberg, C., \& Eriksson, M. (2007). Immediate weight bearing after uncemented total hip arthroplasty with an anteverted stem. Acta Orthopaedica, 76(6), 730-738.

Yeung, E., Jackson, M., Sexton, S., Walter, W., \& Zicat, B. (2011). The effect of obesity on the outcome of hip and knee arthroplasty. International orthopaedics, 35(6), 929-934.

Walker, J. (2009). Osteoarthritis: pathogenesis, clinical features and management. Nursing Standard, 24(1), 35-40.

Walker, J. (2011). Management of osteoarthritis: Jennie Walker presents the strategies available to alleviate patients' joint pain and improve their quality of life. Nursing Older People, 23(9), 14-19. 
Zisberg, A., Shadmi, E., Sinoff, G., Gur-Yaish, N., Srulovici, E., \& Admi, H. (2011). Low mobility during hospitalization and functional decline in older adults. Journal of the American Geriatrics Society, 59(2), 266-273. 


\section{Appendix A}

\section{Data Collection Tool}

\begin{tabular}{|c|c|c|c|c|c|c|c|c|c|c|c|c|c|}
\hline \multicolumn{14}{|c|}{ ANTERIOR } \\
\hline Pt. & Diagnosis & Previous & & & & & & & & & & & \\
\hline$\#$ & of $O A$ & THR & Age & Sex & Height & Weight & BMI & Class & $\begin{array}{c}\text { POD } \\
0\end{array}$ & $\begin{array}{c}\text { POD } \\
1 \\
\end{array}$ & $\begin{array}{c}\text { POD } \\
1 \\
\end{array}$ & $\begin{array}{l}\text { POD } \\
2\end{array}$ & POD2 \\
\hline 1 & & & & & & & & & & & & & \\
\hline 2 & & & & & & & & & & & & & \\
\hline 3 & & & & & & & & & & & & & \\
\hline 4 & & & & & & & & & & & & & \\
\hline 5 & & & & & & & & & & & & & \\
\hline 6 & & & & & & & & & & & & & \\
\hline 7 & & & & & & & & & & & & & \\
\hline 8 & & & & & & & & & & & & & \\
\hline 9 & & & & & & & & & & & & & \\
\hline 10 & & & & & & & & & & & & & \\
\hline 11 & & & & & & & & & & & & & \\
\hline 12 & & & & & & & & & & & & & \\
\hline 13 & & & & & & & & & & & & & \\
\hline 14 & & & & & & & & & & & & & \\
\hline 15 & & & & & & & & & & & & & \\
\hline 16 & & & & & & & & & & & & & \\
\hline 17 & & & & & & & & & & & & & \\
\hline 18 & & & & & & & & & & & & & \\
\hline 19 & & & & & & & & & & & & & \\
\hline 20 & & & & & & & & & & & & & \\
\hline
\end{tabular}




\begin{tabular}{|c|c|c|c|c|c|c|c|c|c|c|c|c|c|}
\hline \multicolumn{14}{|c|}{ POSTERIOR } \\
\hline \multirow{2}{*}{ Pt. } & \multirow{2}{*}{$\begin{array}{c}\text { Diagnosis } \\
\text { of OA }\end{array}$} & \multicolumn{12}{|l|}{ Previous } \\
\hline & & THR & Age & Sex & Height & Weight & BMI & Class & $\begin{array}{c}\text { POD } \\
0\end{array}$ & $\begin{array}{c}\text { POD } \\
1\end{array}$ & $\begin{array}{c}\text { POD } \\
1\end{array}$ & $\begin{array}{c}\text { POD } \\
2\end{array}$ & POD2 \\
\hline \multicolumn{14}{|l|}{1} \\
\hline \multicolumn{14}{|l|}{2} \\
\hline \multicolumn{14}{|l|}{3} \\
\hline \multicolumn{14}{|l|}{4} \\
\hline \multicolumn{14}{|l|}{5} \\
\hline \multicolumn{14}{|l|}{6} \\
\hline \multicolumn{14}{|l|}{7} \\
\hline \multicolumn{14}{|l|}{8} \\
\hline \multicolumn{14}{|l|}{9} \\
\hline \multicolumn{14}{|l|}{10} \\
\hline \multicolumn{14}{|l|}{11} \\
\hline \multicolumn{14}{|l|}{12} \\
\hline \multicolumn{14}{|l|}{13} \\
\hline \multicolumn{14}{|l|}{14} \\
\hline \multicolumn{14}{|l|}{15} \\
\hline \multicolumn{14}{|l|}{16} \\
\hline \multicolumn{14}{|l|}{17} \\
\hline \multicolumn{14}{|l|}{18} \\
\hline \multicolumn{14}{|l|}{19} \\
\hline 20 & & & & & & & & & & & & & \\
\hline
\end{tabular}




\begin{tabular}{|c|c|c|c|c|c|c|c|c|c|c|c|c|c|}
\hline \multicolumn{14}{|c|}{ LATERAL } \\
\hline \multirow{2}{*}{ Pt. } & \multirow{2}{*}{$\begin{array}{c}\text { Diagnosis } \\
\text { of OA }\end{array}$} & \multicolumn{12}{|l|}{ Previous } \\
\hline & & THR & Age & Sex & Height & Weight & BMI & Class & $\begin{array}{c}\text { POD } \\
0\end{array}$ & $\begin{array}{c}\text { POD } \\
1\end{array}$ & $\begin{array}{c}\text { POD } \\
1\end{array}$ & $\begin{array}{c}\text { POD } \\
2\end{array}$ & POD2 \\
\hline \multicolumn{14}{|l|}{1} \\
\hline \multicolumn{14}{|l|}{2} \\
\hline \multicolumn{14}{|l|}{3} \\
\hline \multicolumn{14}{|l|}{4} \\
\hline \multicolumn{14}{|l|}{5} \\
\hline \multicolumn{14}{|l|}{6} \\
\hline \multicolumn{14}{|l|}{7} \\
\hline \multicolumn{14}{|l|}{8} \\
\hline \multicolumn{14}{|l|}{9} \\
\hline \multicolumn{14}{|l|}{10} \\
\hline \multicolumn{14}{|l|}{11} \\
\hline \multicolumn{14}{|l|}{12} \\
\hline \multicolumn{14}{|l|}{13} \\
\hline \multicolumn{14}{|l|}{14} \\
\hline \multicolumn{14}{|l|}{15} \\
\hline \multicolumn{14}{|l|}{16} \\
\hline \multicolumn{14}{|l|}{17} \\
\hline \multicolumn{14}{|l|}{18} \\
\hline \multicolumn{14}{|l|}{19} \\
\hline 20 & & & & & & & & & & & & & \\
\hline
\end{tabular}

